\title{
La apuesta científica y
} humanista de la Universidad de Salamanca. (Entrevista con el Rector de Salamanca)

\author{
Santiago Centeno Richardson; Marcela Soledad Guerrero \\ Segovia y Rigliana Ximena Portugal Escóbar
}

Arbor CLXXIII, 683-684 (Noviembre-Diciembre 2002), 669-689 pp.

Bajo la mirada crítica de su rector, repasamos los aspectos más relevantes de una de las Universidades más prestigiosas y antiguas del mundo que, con la entrada al nuevo milenio, expande su contribución a la educación y proyecta aún más su proyección innovadora.

Después de dos períodos consecutivos como Rector, Ignacio Berdugo Gómez de la Torre describe, desde su óptica, el lugar que ocupa la Universidad de Salamanca en el panorama internacional. Considera prioritario e imprescindible buscar la convergencia de quienes son actores responsables en la formación de profesionales, las universidades y las administraciones.

Consciente de la importancia histórica de la USAL tanto en Europa como en Latinoamérica, siendo para esta última una referencia indiscutida, Berdugo responde cómo concibe la institución el hecho de ser una fuente de producción de dos culturas: la tradicionalmente humanista y la cada vez más pujante cultura científica. 


\section{Santiago Centeno, Marcela Guerrero y Rigliana Portugal}

\section{Introducción}

En una sociedad globalizada donde el conocimiento se constituye en el recurso de mayor valor, la Universidad de Salamanca proyecta su accionar con responsabilidad frente a la sociedad en la que se inserta y de la cual también se nutre. Se apunta, en esta interacción, a generar mecanismos que posibiliten, entre otros, lograr mayor capacidad científica e iniciativa cultural, en la búsqueda permanente de excelencia académica.

La USAL extiende su actividad académica y científica al ámbito mundial, con especial atención a Iberoamérica y Europa. Reflejado claramente en la concurrencia de alumnos americanos que cada vez se incrementa más, como señalan los indicadores académicos en los últimos años, a la vez que se consolida el espacio común de la enseñanza superior planteado como premisa a nivel de la Comunidad Europea.

La Universidad, fundada en 1218, se mantiene aún como referente en materia de producción del saber, y es esta trascendencia dentro del continente lo que la hace ser de manera constante sede de decisiones de amplio horizonte cultural. Tal el caso del Tribunal Penal Internacional, que se gestó en sus aulas en el siglo XVI o más recientemente, en 2001, en el marco de la Convención de Salamanca, la creación de una Asociación Europea de la Universidad, para propiciar un espacio cada vez más vasto de intercambio de profesionales y alumnos entre naciones.

Asimismo, Salamanca destaca como sede donde se creó la Conferencia de Rectores de Universidades Españolas, entidad que proyecta el acercamiento de opiniones, del entorno académico, al ámbito de la Administración. En un momento como el actual, donde las instituciones de educación superior deben adecuarse a un nuevo marco de la Ley Orgánica de Universidades (LOU), acogido conflictivamente, el Rector de esta casa de estudios, Ignacio Berdugo, confía en que su presidencia en la CRUE pueda ubicar a la Universidad de Salamanca en una posición de liderazgo dentro del sistema universitario.

Finalmente, se desencadena en el análisis de las dos culturas que alimentan a la USAL: la tradicionalmente humanista y la científica. Precisando Berdugo la inconveniencia y falta de criterio de quienes establecen el versus entre una y otra o la demarcación rígida de fronteras entre lo científico y lo humanista. "Abraham Zacut fue un sabio de su tiempo, un humanista de su tiempo que, junto a conocimientos de física tenía conocimientos que hoy calificaríamos de estrictamente hu- 


\section{La apuesta científica..., entrevista con el Rector}

manistas, a lo mejor porque la frontera de los saberes no es tan delimitada como pretendemos».

"Tal vez donde resulta más complejo llevar a cabo la internacionalización es en la enseñanza de pregrado y en la formación de profesionales, porque ambas están vinculadas a la propia historia de las profesiones en cada país y a la movilidad o no de profesionales entre Estados».

\section{¿Existe promoción de la Universidad en el ámbito mundial?}

En la última década se ha establecido una promoción que busca la internacionalización de esta universidad. Por un lado aparece casi como una necesidad política, puesto que el mundo se regionaliza. Pero además existen otros ámbitos ligados a áreas culturales, tal el caso del espacio iberoamericano que tiene una dimensión política, pero también cultural.

Se busca dar consistencia a las relaciones interestatales, a la comunidad de Estados. Hay que buscar detrás también una comunidad cultural y una comunidad de enseñanza. De hecho, se puede decir que la comunidad política es una consecuencia de una serie de condiciones previas entre las cuales está la cultural.

Pero, aparte de estos hechos, hay otro elemento de internacionalización que siempre se ha producido, como es el de la investigación. Allí, sin necesidad de más pasos, es donde primero se ha dado éste proceso.

Por otro lado, tal vez donde resulta más complejo llevar a cabo la internacionalización es en la enseñanza de pregrado y en la formación de profesionales, porque ambas áreas están vinculadas a la propia historia de las profesiones en cada país y a la movilidad o no de profesionales entre Estados. Asimismo, entran en juego intereses o factores que no son estrictamente universitarios, son extrauniversitarios, pero que condicionan su posibilidad real. En ese camino se ha trabajado mucho durante los últimos treinta años en Europa, todo el tema de la movilidad de estudiantes, es un camino clave que persigue con énfasis la propia Comunidad. Es un modo de actuar o un modelo que se está trasladando también a otras regiones del mundo donde siempre existe esa cercanía entre Estados, de la que se habló anteriormente, aproximación que requiere también de esa relación cultural, dentro la cual se inserta la actividad universitaria. 


\section{¿Hay alguna herramienta concreta para fomentar la internacionalización en ese plano más difícil del pregrado y la formación de profesionales?}

En la Universidad confluyen las responsabilidades de varias instituciones. Evidentemente una es la universitaria, pero también converge la propia responsabilidad de la Administración. Es decir que la regulación de las competencias profesionales y las profesiones resulta de un terreno compartido entre la Universidad, que es quien pone los contenidos, y paralelamente lo que espera de ella la sociedad a través de la Administración.

Es evidente que la Administración es quien regula los requisitos de formación de un médico, un abogado o un ingeniero pero, por otra parte, es la universidad la que establece los contenidos concretos a ser impartidos. En síntesis, es una convergencia de intereses.

Viendo el tema desde la parte de la Universidad, es posible inferir que hay otros factores que le conciernen a ésta y que son decisiones de mediano y largo plazo. Se considera el crear condiciones para que los estudiantes conozcan la realidad de los países, que la conozcan universitariamente. Por ejemplo, si en Salamanca hay un Centro Cultural Hispano-Japonés ésa es una oportunidad para que los estudiantes españoles tengan acceso a una cultura y una realidad que no van a percibir normalmente en las aulas. Lo mismo ocurriría con el Centro de Estudios Brasileños o el Centro Hispano-Británico. Es necesario generar estructuras dentro de la Universidad que actúen como un factor que potencie esa internacionalidad.

«...la cultura de la movilidad estudiantil no es tan sencilla, por este motivo es una cultura que debe irse cultivando y fomentando".

\section{Dentro de la vocación internacional de la USAL ¿es prioritario el intercambio simétrico de alumnos y profesores?}

El tema del intercambio simétrico está en tener la opción, otra cosa es que se use. En este momento la realidad es que la universidad recibe mucha más gente de la que envía. Está mucho más definida esta política de recibir que de enviar porque está más potenciada económicamente. Hay becas para venir a Salamanca, pero son mucho menores las becas para que estudiantes españoles vayan afuera. Prácticamente están vigentes sólo los intercambios y becas que tiene la Universidad, además del programa Sócrates, que financia (simbólica- 
La apuesta científica..., entrevista con el Rector

mente) la Unión Europea y el programa Intercampus que, respecto a América Latina, en este momento es en absoluto residual numéricamente.

A pesar de lo anterior, existe un programa específico que da posibilidades a que el intercambio sea simétrico, es un programa bilateral. $\mathrm{Si}$ se ofrecen cien plazas para que vengan estudiantes de otras universidades a Salamanca, hay cien oportunidades para que estudiantes nuestros vayan afuera. Pero, eso es lo que proponemos y la realidad es que la cultura de la movilidad estudiantil no es tan sencilla, por este motivo es una cultura que debe irse cultivando y fomentando. Por ahora no se puede hablar de igualdad y como ejemplo está el programa Erasmus, a través del cual recibimos mil estudiantes y enviamos quinientos. Como se puede ver, la USAL cubre la mitad de las plazas que oferta.

\section{Esto es sólo a nivel de pregrado. ¿Qué sucede con los postgrados?}

En el ámbito de los postgrados es distinto. Lo que hay en este nivel son relaciones bilaterales. Hay una recepción muy importante de alumnos latinoamericanos en doctorado. El 40 por cien proviene de allí, pero la relación en este caso no necesariamente tiene que ser desigual, porque va a depender de la oferta de esa formación de postgrado que exista en otros países.

Los estudiantes españoles en postgrado normalmente reciben esta formación en Estados Unidos o en países centroeuropeos; a veces también hay estancias cortas en América Latina, fundamentalmente en México y Brasil. Otra posibilidad es la de estancias de investigadores españoles en Latinoamérica, pero como producto de un proyecto de investigación gestionado desde aquí.

En la Convención de Instituciones Europeas de Enseñanza Superior, realizada en Salamanca, se reunieron más de trescientos organismos representativos en la educación superior, para preparar su aporte a la Conferencia de Ministros responsables de los paises firmantes de la Declaración de Bolonia.

En el ámbito de la Convención se fusionaron las dos grandes asociaciones de universidades que existían en toda Europa. Una tenía como socios fundamentales a las asociaciones de universidades de cada país y la otra a las universidades individualmente. En ese marco se reiteró el compromiso de crear, antes de finalizado el decenio, un Espacio Europeo de la Enseñanza Superior, manteniendo los principios de la Declaración de Bolonia. 


\section{¿Qué trascendencia tuvo la Convención de Instituciones Europeas de Enseñanza Superior, realizada a finales de marzo de 2001 en Salamanca?}

Esta convención fue importante en la medida que, como resultado de esas sesiones, se conformó una nueva estructura, la Asociación Europea de la Universidad. Esta entidad hará de interlocutor ante la Unión Europea acerca de las decisiones que se adopten en el marco comunitario, respecto a la creación de un Espacio Europeo de la Enseñanza Superior. Para entender, digamos que es la CRUE europea; es a quien se va a pedir la opinión respecto a las decisiones que la Unión adopte en temas como, por ejemplo, la convergencia en materia de titulaciones.

\section{Dentro de las reuniones a nivel comunitario, ¿el tema más relevante es el sistema de créditos común para las titulaciones europeas?}

No, lo que se discute está vinculado a otras decisiones, es decir, surge la pregunta del por qué es necesario que converjan los sistema universitarios. Y la respuesta es directa, para que se garantice la movilidad de profesionales. Si la formación de un médico fuera distinta en Bélgica y en Alemania, pues, difícilmente podría aceptarse el intercambio de profesionales. El esquema es ése: hacer un catálogo común de estudios, respetando particularidades, lo que se plantea aquí es trasladar esa estructura coherente para todos los países europeos. Deben respetarse los márgenes de autonomía de cada país y de cada universidad en concreto, es decir, que se mantenga el perfil que tiene una titulación de un lugar respecto a otro, manteniendo el tronco común.

En esta línea de unificación de criterios destaca a principios de los 80 una serie de directivas europeas respecto a los títulos biosanitarios. Ahora se pretende que esto sea análogo para otras titulaciones y lo mismo respecto a doctorados, lo que pasa es que el proceso lleva debatiéndose más de veinte años.

\section{¿En este proceso hay un espacio de inserción para Iberoamérica?}

Es una segunda fase donde creo que España y Portugal pueden tener un papel importante porque, de una forma $u$ otra, gran parte 
La apuesta científica..., entrevista con el Rector

de la estructura de las titulaciones en Iberoamérica son próximas a las que se dan en la península Ibérica; ése paso es mucho más fácil.

Aunque cabe suponer lo contrario, es mucho más diversa históricamente la formación dentro de Europa, porque son sistemas universitarios con menos comunicaciones que las históricamente mantenidas en el ámbito iberoamericano. Claro que la relación en este último espacio se facilita por el tema de la lengua.

La Universidad de Salamanca dentro de su oferta académica comprende en el curso 2000-2001 a nivel de pregrado, 72 carreras $y$ en postgrado 103 estudios. En el mismo curso académico se expidieron 168 Titulos de Doctor, 747 Titulos Propios, 2280 de Licenciado y 1835 de Diplomado.

En 1998 consideraba que las titulaciones ofrecidas por la USAL eran suficientes y que, en todo caso, había que afianzarlas en calidad, ¿cree que en este momento es necesario reducir o aumentar la oferta académica?

La oferta de titulaciones no va a crecer. La USAL es la institución en España que más titulaciones ofrece y si quedan cosas por hacer, son temas absolutamente residuales. Por ejemplo, este año se ha creado una titulación de Estudios de Asia Oriental y además la Universidad tiene un Centro de Estudios Japoneses, pero esto ya es un detalle, porque la gran estructura de titulaciones está. Lo que se debe hacer ahora es trabajar con los departamentos que hay, para mejorarlos en sus medios y sus posibilidades, para dispensar una mejor función, tanto docente como investigadora.

\section{¿Son los cambios científico-tecnológicos una de las principales causas por las que se observa la constante demanda de formación de postgrado? ¿Existen otros motivos para esta demanda?}

Para contestar esta pregunta, podemos referir que se presentan dos causas. La primera está vinculada de alguna manera a lo que es formación permanente, formación continua. Uno se forma de determinada manera, con medios establecidos y percepciones. Los conocimientos cambian en la especialidad que a uno le interesa, por el propio desarrollo de la ciencia en los últimos tiempos, ante lo cual uno busca actualizarse. ¿Cómo responde la universidad a esa nueva exigencia de formación? La respuesta es que depende de la importancia 


\section{Santiago Centeno, Marcela Guerrero y Rigliana Portugal}

del curso o del tipo de destinatario; también depende del contacto que entable la Universidad con otras instancias que puedan colaborar en esta formación.

Paralelamente, hay otro dato que tampoco conviene olvidar o desechar, que es el crecimiento horizontal de la población universitaria en España, no tanto numéricamente como proporcionalmente. El número de estudiantes españoles que estando en edad de cursar estudios superiores, y lo hacen, resulta muy elevado; en Europa ocupamos uno de los primeros lugares. Esto supone el tener que introducir elementos de cualificación añadidos que permitan entrar luego al mercado de trabajo, para ser más competitivos. Entonces, la mayoría busca tener un postgrado, que sea un plus sobre el título obtenido en la Universidad.

\section{En ese sentido, en la Convención de Salamanca se apunta a encarar dos tipos de estudios: uno que desemboque en salidas laborales rápidas y otro que conduzca al posgrado ¿Cuál es su criterio?}

Puede que ésa sea un poco la estructura, pero al respecto el elemento más claro es Latinoamérica. En muchos países latinoamericanos el postgrado cumple una función diferenciadora en relación a la formación previa. Ante el crecimiento horizontal de un sistema, es propio que se introduzcan elementos que marquen la diferencia. Sin embargo, este panorama latinoamericano puede no ser trasladable a Europa aunque, en última instancia ésos son los parámetros cuando se está frente al crecimiento horizontal. Por lo señalado, los factores convergen, por un lado más información permanente, expresada en cursos de actualización, etcétera y, por otro lado, postgrados concretos, que tienen que ver con una mejor cualificación en el mercado de trabajo.

«...la raíz última de todo el sistema universitario latinoamericano es la USAL».

¿Cuál de sus actividades le han permitido relacionarse con mayor fluidez con los institutos y universidades latinoamericanas, la desplegada como profesional en Derecho o como Rector?

Antes de ser Rector ya mantenía una relación personal con diversas instituciones, viajé a América en junio de 1986 y, desde entonces, los 
La apuesta científica..., entrevista con el Rector

vínculos se han ido afianzando. Además, a nivel académico, continuamente he trabajado con alumnos de doctorado procedentes de América Latina.

Sin embargo, creo que hay algo que ha favorecido mucho más la relación, un elemento clave, que es la proyección histórica que Salamanca tiene como Universidad en Latinoamérica, con lo cual ser rector de la USAL supone una relevante tarjeta de presentación. A esto hay que sumar el trabajo de equipo en la gestión rectoral, combinado con la cobertura de los medios de comunicación y la rapidez de las comunicaciones en general, factores que favorecen más las relaciones que había antes.

\section{¿Por qué es tan buena carta de presentación ser rector de la Universidad de Salamanca?}

Porque la raíz última de todo el sistema universitario latinoamericano es la Universidad de Salamanca. Recordemos que las universidades fundadas en las colonias americanas, se erigían proyectando algunos privilegios propios de la Universidad de Salamanca. Ésta representaba una referencia idealizada de la formación universitaria y eso se mantiene, es una ley que está contenida en la frase «lo que la Naturaleza no da, Salamanca no lo presta». Donde más he escuchado este dicho popular es en América, no aquí, y esto muestra el significado que entraña Salamanca para los latinoamericanos.

De su trayectoria destaca una relación nutrida con institutos y universidades de Perú y Colombia ¿tienen estas casas de estudio algo en común con la de Salamanca, que no tengan otras universidades latinoamericanas?

La relación existente es igual. En forma particular comencé vinculándome primero con Chile y más tarde con Perú y Colombia. Pero es importante señalar que históricamente Salamanca mantuvo relación, desde la colonia, con la Universidad de San Marcos de Lima y la UNAM.

$\mathrm{Al}$ presente, la USAL se relaciona de manera óptima con México y Brasil, donde se está estudiando la posibilidad de establecer las próximas sedes, al igual que las existentes en Colombia y Argentina. 


\section{Santiago Centeno, Marcela Guerrero y Rigliana Portugal}

Quien deba asumir el próximo período rectoral, desde 2003, tendrá que programar su gestión adaptándola a los nuevos marcos que establece La Ley Orgánica de Universidades (LOU), publicada en el BOE el 24 de diciembre de 2001 y en vigor desde el 14 de enero de este año. A esta nueva disposición se le acusa, entre otros reclamos, de no fijar sus metas hacia una universidad pública, crítica, científica y de calidad. Lo concerniente a la autonomía universitaria se ha vuelto uno de los puntos más debatidos en diversos medios de comunicación nacionales.

La normativa que dispone la presencia de miembros del Consejo Social en el Consejo de Gobierno de las universidades, ha encontrado la oposición de colectivos estudiantiles y académicos, por considerar que esto implica un manejo político de la autonomía. La Conferencia de Rectores de Universidades Españolas (CRUE) manifestó su discrepancia respecto a la LOU desde la presentación del proyecto. A través de su Asamblea la CRUE opinó que el nuevo marco «afectaría gravemente a la capacidad de las instituciones universitarias para adaptarse a un contexto y demandas continuamente cambiantes y garantizar la calidad del servicio público de la educación superior».

\section{¿Cómo evalúa el «proyecto universitario razonado», planteado en su candidatura de 1998, como una forma de coordinar adecuadamente la educación superior y el Estado?}

La coordinación entre la educación superior y el Estado ha tenido algún tipo de crisis importante en el curso pasado. Todo lo que fue el debate de la Ley Orgánica de Universidades. Creo que, como he comentado antes, sobre el hecho educativo hay responsabilidades plurales que deben ser asumidas; que a mí me importan y creo que es razonable que la Administración tenga sus responsabilidades respecto a la propia Universidad. De ahí la tensión que siempre se ha generado entre la autonomía universitaria y la responsabilidad de la Administración.

El año pasado, en ése sentido, no fue un buen año - no voy a entrar ahora a valorar las causas o atribuir responsabilidades-. No lo fue en cuanto se produjo una falta de comunicación evidente entre el sistema universitario, las universidades en su conjunto, y la Administración. Pero la Administración educativa a nivel estatal, no tanto a nivel de comunidades autónomas; porque respecto a nuestra comunidad autónoma la relación siempre ha sido fluida y no ha existido tal incomunicación. A lo mejor porque al ser menor el número de interlocutores es más fácil. 
La apuesta científica..., entrevista con el Rector

Pienso que en aquel momento fue prioritario cumplir un calendario, por sobre el método de trabajo. Tendría que haberse dado un mayor grado de acuerdo en cuanto a la manera de trabajar; precisamente lo que no hubo fue acuerdo y eso ha lastrado las universidades. No es que las universidades no lo acatemos, ¿cómo no lo vamos a hacer si está absolutamente legitimado? Lo que pasa es que faltó la oportunidad política de cómo hacerlo. Opino que se han reformado cosas que nadie demandó que se reformaran. Pero bueno, ahí está y ahora lo que hay que hacer es intentar desarrollar la aplicación de esa ley, de acuerdo con el mayor grado de respeto a la autonomía de las universidades. Creo que se ha mejorado algo y va a mejorar más.

Por su parte el Ministerio de Educación debe crear una convergencia y abrir un proceso de diálogo entre todos los actores implicados, para que cada uno, desde sus responsabilidades, pueda llegar a soluciones lo más consensuadas posible en normativas de desarrollo. Hay temas tan importantes como la habilitación o como es el doctorado, que están ahí, en la puerta y que deben regularse desde los próximos meses.

\section{La Ley Orgánica de Universidades tuvo baja aceptación o ninguna en los claustros universitarios, ¿va a dificultarse el desenvolvimiento de la USAL y las demás universidades españolas en este nuevo marco orgánico?}

Hubo un momento, que todavía estamos viviendo, de estar haciendo cosas que no tenemos necesidad de hacer. Vamos a ver, se ha entrado en un proceso de dos largos años - por lo menos- en todas las universidades españolas, de empezar a tomar todo tipo de medidas para adecuarse a la nueva normativa y eso hace que la Universidad mire mucho hacia adentro; lo cual es positivo, a veces, pero pienso que ahora puede que esté mirándose demasiado. Esto hace, en definitiva, que elementos de gestión, de gestión de investigación, de docencia y de relaciones internacionales ahora se vean lastrados por un tema de tiempo. Así, la propia comunidad universitaria va a estar aplicando un modelo que puede funcionar o no, cuando había temas evidentemente estabilizados y nadie pedía que se cambiaran.

Realmente, si nos fijamos en los últimos diez años lo que se debatía era la necesidad de reformar, sobre todo, el sistema de acceso del profesorado, lo que era el personal académico, pero al final se reformó todo. 


\section{¿En qué sentido la LOU obstaculiza la autonomía universitaria, tal como ha sostenido la CRUE?}

No querría volver a abrir un tema que ya está tratado. Hablo ahora como jurista -que creo que soy todavía-. Un derecho, como es la autonomía universitaria, puede limitarse más o menos. Considero que necesariamente cualquier derecho es limitado. No hay ni un solo derecho ilimitado, ni siquiera la vida. Entonces, pues, la Ley Orgánica de Universidades, limita más la autonomía universitaria que lo que la limitaba la Ley de Reforma Universitaria; ése es el punto clave.

Cabe preguntarse, luego, si es contraria a la autonomía universitaria. Hay temas que están siendo analizados en este momento por el Tribunal Constitucional, ya veremos qué sucede. Hay temas de selección de profesorado que son más que discutibles desde el punto de vista de la autonomía universitaria; que hay cuestiones de presencia de miembros del Consejo Social, dentro de los órganos de gobierno de la universidad, que también son discutibles. Como todo en Derecho puede ser opinable, desde mi opinión yo creo que en torno a estos puntos ha habido limitación, al menos una mayor de la que había con la Ley de Reforma.

\section{¿En qué medida cree que su participación en la CRUE ha beneficiado su gestión en la Universidad de Salamanca?}

Estimo que deben fomentarse siempre los instrumentos de cohesión en el sistema universitario y la CRUE es un instrumento generado por las universidades para coordinar este sistema. El que esté el rector de Salamanca allí y que esté implicado y sea hasta este momento el presidente de la CRUE es positivo para la institución, porque supone un elemento de liderazgo de la Universidad de Salamanca respecto al sistema universitario español. Creo que eso beneficia siempre en política exterior e interior a esta universidad.

\section{Desde su gestión en 1998 como vicepresidente de la CRUE ¿hay algo que haya quedado pendiente y que pueda concretar ahora que asumió la presidencia?}

Considero que hay algo pendiente en el aspecto organizativo, a pesar de que llevamos años trabajando en ello. Para comprender esto 
es importante recordar que la CRUE nació en Salamanca -justamente el año próximo cumplirá un decenio- y comenzó con una estructura incipiente, limitando su accionar al de una asamblea de universidades. Al presente sigue trabajando de esta manera, sin embargo, ahora requiere decisiones de funcionalidad, teniendo en cuenta además el crecimiento del sistema universitario español. Todavía se mantiene el formato anterior, según el cual las universidades se reunían por materias; por ejemplo, había una comisión donde estaban todos los vicerrectores de relaciones internacionales de las universidades españolas, otra con los gerentes, etcétera.

Lo que se requiere, entonces, es una respuesta estructural, ya que en este momento hay por lo menos diez subcomisiones que dependen de una forma u otra de la CRUE. Dentro de esta reforma de estructura debe potenciarse, además, la parte administrativa e incluso inmobiliaria de la propia comisión de rectores. En eso estamos, yo creo que sería el primer punto que ha quedado pendiente, teniendo en cuenta que lleva años esperando recibir una respuesta cerrada y eficaz.

\section{Con respecto al proyecto académico que usted definió para su gestión rectoral, ¿considera que éste responde a la demanda social actual?}

En este sentido opino que hay que revisar el proyecto, debido a que un plan que se diseña en un programa electoral marca grandes líneas de actuación, que luego muchas veces deben adaptarse a una realidad que cambia, pero en líneas generales se ha cumplido. Había un condicionante, que era toda la primera parte, la referida a lo que se ha producido en este año último con el tema de la Ley Orgánica de Universidades. Ahora hay que revisarlo.

\section{¿Por qué postularse nuevamente para Rector?}

Siempre hay algo que queda pendiente, pero es inevitable. Me parece y creo que, sin prestarse a malas interpretaciones, es un momento de cambios dentro de la universidad española, incluyendo, por supuesto, a la Universidad de Salamanca. Aquí, en esta coyuntura, aunque pueda haber gente que discrepe con lo que digo, nuestra candidatura es una candidatura que tiene experiencia en gestión; permite, en momento 
de inestabilidad o de posible inestabilidad, darle estabilidad a la vida universitaria, pero finalmente quienes deciden son los votantes.

\section{¿Cuáles son los temas prioritarios que consideraría, de ser reelegido como primera autoridad universitaria?}

Si tuviera que priorizar las tareas para los próximos cuatro años, desde luego, en primer lugar, habría que poner sobre la mesa el resultado de todo el proceso de evaluación institucional que se llevó a cabo a lo largo de este período y proyectarlo, de manera global, en medidas concretas respecto a la gestión de la docencia, investigación y la propia gestión universitaria.

Hay una segunda parte que tampoco puede obviarse y es la de tomar decisiones al hilo de lo que están tomando en toda España respecto a la conversación europea, donde tampoco puedes perder el tren ahí. Tienes que amoldar tus estructuras para poder dar una respuesta ágil.

Una tercera parte va vinculada a todo lo que es internacionalización y nuevas tecnologías. Yo creo que hay una respuesta que debemos dar. Que no sabes en este momento, tampoco, cuál es el límite, porque de nuevas tecnologías todos hablamos pero a lo mejor lo que digamos hoy dentro de cuatro años tenemos que revisarlo, porque la tecnología ha ido más adelante de lo que pensábamos.

\section{¿Cuáles son las necesidades a ser subsanadas en caso de una reelección?}

En primer lugar es necesario no tener límite en el número de vicerrectores. En el Estatuto dice que son seis los vicerrectores y eso es disfuncional, porque se requiere que existan dos más, uno que pueda mirar el tema de gestión de calidad y otro que trabaje con innovación y nuevas tecnologías.

En segundo lugar apremia la puesta en marcha de la reorganización de universidades, que es necesaria y que es justamente todo el tema de las políticas de profesorado. Tomemos en cuenta que la actual política de profesorado está marcada por dos lados, por una parte los funcionarios y por otra los números o las cantidades de plantillas que se definen en función de la necesidad docente, ésos son los dos parámetros hasta ahora vigentes. Sin embargo, la Ley Orgánica de Universidades ofrece 
la norma de desarrollo que ha gestionado la Junta de Castilla y León y que presentan otras opciones. Se introduce, por ejemplo, la opción contrato, pero contrato estable, no por un corto período de tiempo, sino contrato permanente.

\section{¿Cuál el vínculo entre la USAL y el Tribunal Penal Internacional?}

El Tribunal Internacional que se ha puesto en marcha ahora, tuvo una etapa preparatoria aquí en Salamanca. Todo lo que es la proyección internacional del Derecho se empieza a gestionar en el $s$ XVI en estas aulas, se inicia la construcción teórica. Esos son símbolos en la Universidad y por tanto hay que cuidarlos, ya que tienen un significado, y es importante mantenerlos. No debe la Universidad renunciar a sus símbolos.

A pesar de haber transcurrido más de ocho siglos desde su formación, la Universidad de Salamanca está elaborando una nueva etapa en la cual busca acrecentar la presencia científica en sus aulas, de manera que exista una presencia equitativa entre las dos culturas. La primera humanista y famosa por personajes como Fray Luis de León o Unamuno; la segunda busca su reconocimiento a través de proyectos de desarrollo científico como el CIC.

Ante esta situación, el rector refiere la actualización en los objetivos de esta casa de estudios, generados principalmente por un cambio interno que busca ir más allá de generar saber y transmitirlo. Para Berdugo es evidente que, ni las condiciones ni el objetivo específico, han sido los mismos en cada momento histórico; ni el contenido de las cosas que se han enseñado en diferentes etapas se ha repetido. No obstante, a veces, la falta de adecuación a las nuevas necesidades o realidades, ha motivado las crisis de la Universidad a través de su historia. No hay que olvidar el conflicto acaecido durante el siglo XVIII, donde todavía se seguía transmitiendo un saber que no correspondía a las exigencias que mantenía la sociedad en aquel momento. En nuestros dias, la Universidad sigue generando ese saber y transmitiéndolo a sus alumnos y comunidad académica, a través de una serie de programas puestos en marcha.

\section{¿Piensa que Salamanca puede considerarse cuna de intelectuales humanistas y al mismo tiempo qué pasa con la apuesta científica en este sentido?}

Nadie discute que el humanismo esté vinculado a esta Universidad, desde los grandes literatos españoles, que estudiaron la lengua hasta, 
en un sentido más amplio, aquellos que estudiaron el Derecho, etc. Pero, en la historia de esta universidad podemos encontrar momentos donde esa dicotomía, de pretender ver a la ciencia versus el humanismo, no se daba y es aconsejable que no se diera. Podemos dar un ejemplo conocido por todos, refiriéndonos a Abraham Zacut. Él fue un sabio de su tiempo, un humanista que además de sus estudios en física, poseía conocimientos que hoy calificaríamos de estrictamente humanistas, tal vez porque la frontera de los saberes no es tan delimitada como pretendemos. Por otro lado, hace un mes se entregó el Honoris Causa en esta Universidad a George Steiner que es considerado por todos como un humanista. Steiner reivindicaba y reclamaba que un humanista no debe desechar, sino tender a incorporar también una formación en ciencias. Él es un físico que ahora hace humanidades - crítica literaria-, como si fuera algo distinto, pero no es así.

Dentro de lo que son los parámetros actuales del saber, donde, efectivamente, está más compartamentalizado lo que estaba hace siglos o hace años, el perfil de la Universidad de Salamanca no es solamente de Letras y Humanidades.

\section{Si planteamos a las ciencias y a las humanidades como dos culturas, ¿se puede hablar de predominio de una sobre la otra?}

En cada momento histórico son las personas, en sus respectivos departamentos, las que finalmente dan un perfil a las distintas etapas de la Universidad. Por ejemplo, si tocara hablar de la identidad de esta casa de estudios, se debería mencionar al Centro de Investigación del Cáncer. Pero ¿por eso deja de tener interés la investigación de la lengua en la Universidad de Salamanca o deja de ser puntera? La respuesta es no.

\section{¿Cuáles son las principales áreas de investigación en ciencias que está desarrollando la universidad?}

Existe una línea genérica grande en el ámbito de la investigación biosanitaria, donde hay en este momento tres institutos de investigación: uno del cáncer, otro de bioquímica y biología y un tercero de neurología. Surgen centros cuando hay personas capacitadas para integrarlo. De hecho, en este momento se está comenzando a montar la estructura 
de un centro de investigaciones en el ámbito agrario y el tema de genética vinculado a lo que es el estudio y desarrollo del campo en esta zona. En esta última área están también las aportaciones de las facultades de Ciencia, Biología y Química a través de sus departamentos docentes.

Por otro lado, a la hora de percibir el asunto en cuanto a proyectos de investigación, se puede identificar como un signo clave el bloque de lo biosanitario, pero esto puede ser debido al desarrollo que esta área tiene dentro del país, en general. Es un investigación puntera.

\section{¿Cuál es el papel que está desempeñando el Centro de Investigación del Cáncer (CIC)?}

El Centro de Investigación del Cáncer es clave por dos razones: la primera es por la calidad de las personas que hay trabajando en él; la segunda es la trascendencia de la investigación que realiza. Pero sobre todo, porque es un modelo de gestión. Allí se incorpora personal que está periódicamente sometido a evaluación externa, mientras haya algún proyecto, lo que constituye un acicate para el propio centro.

Es posible referir que este es un modelo no agotable, es un proyecto en crecimiento. Como ejemplo de lo anterior se puede decir que no todas las instalaciones del centro están en este momento ocupadas, sino que gracias al propio perfil que van a ir tomando las investigaciones se determinará su nivel de ocupación. Otro elemento que es clave es también para el desarrollo de la investigación y para la continuidad del Centro es la construcción del animalario, que va a servir para todo el campus universitario.

El CIC representa otra vertiente importante ha destacar; es un lugar de encuentro entre investigación básica y aplicada. Esta última tanto desde el punto de vista clínico como de los grupos de investigadores más relacionados con el mundo de la industria farmacéutica.

Desde el punto de vista arquitectónico, el centro fue diseñado en contacto directo con los científicos y el equipo que lo construyó. A la hora de levantar un edificio dedicado a la enseñanza o la investigación siempre está la «tensión», entre los usuarios y el que lo diseña. Acá hubo un método de trabajo un poco especial por el que cada vez que se definía cómo sería un espacio en concreto, el responsable tenía que dar su conformidad por escrito, con lo que se podía avanzar. El resultado de este trabajo fue muy positivo porque se llegó a un muy buen diálogo entre los dos equipos participantes. 


\section{Santiago Centeno, Marcela Guerrero y Rigliana Portugal}

¿Se puede decir que la Universidad de Salamanca está orientada hacia el desarrollo tecnológico, y de ser así, cómo se explica la contradicción que podría existir cuando se asume que Castilla y León no constituye un foco industrial?

Para hablar de ello es casi como entrar en un círculo vicioso, un círculo diabólico. ¿Qué es primero, el huevo o la gallina? No hay desarrollo tecnológico porque no hay industria; no hay industria porque la universidad no provee ingenieros para que haya industria. Hay que tomar decisiones estratégicas donde -como se refería antes- exista convergencia entre las decisiones de la administración y de la propia universidad. Ése es, un poco, el marco general. Dada esta situación, ¿cómo puede potenciar la universidad el trabajo industrial?. Salamanca no es un foco industrial, sin duda. No obstante, - volviendo al principioexisten departamentos del ámbito biosanitario. Desde allí se ha trabajado en los últimos diez años, generando pequeñas industrias. Es una línea que hay que fomentar, al igual que el programa Galileo cuyo fin es poner en marcha la creación de empresas que incentiven esa área.

Lo «industrial» de Salamanca es la lengua. De hecho la ciudad tiene una sociedad que mueve mil millones al año. No está mal, pero la industria de la lengua genera una actividad en torno a ella que incluye el desarrollo de materiales para su enseñanza, tanto en soporte bibliográfico como informático. La industria cultural ya se está fomentando desde la universidad. Pero también está, desde fuera, la otra industria, la clásica, por ejemplo la industria química o la eléctrica, donde los pasos son más lentos y donde la universidad se limita solamente a ser socio externo; pero fomentando solamente a ésa escala.

\section{¿Se puede hablar de una identidad de la USAL, más ligada a lo humanista, y mantenida como símbolo aunque el desarrollo de la región vaya por otras vertientes?}

En absoluto. Sobre ese ámbito -que son los activos- hay que apostar a la transformación en riqueza y en desarrollo hacia la sociedad, considerando que es aquello en que se es más fuertes. ¿Cuáles son estas áreas de investigación en la Universidad? Pues sin duda, la lengua, pero también el ámbito biosanitario, y cuando esto es detectado se trabaja en ello. 
La apuesta científica..., entrevista con el Rector

\begin{abstract}
¿Cuál de las tres amplias áreas de la universidad -docencia, investigación y extensión- ha tenido mayor despegue durante su gestión?
\end{abstract}

Sin duda la investigación es el área más desarrollada, debido principalmente a que se cuenta con los centros. Lo que sucede es que a veces es muy difícil separar la investigación de la docencia. En la universidad existe una docencia que es tributaria directa, y que trabaja en unión directa con la investigación que se realiza. Además el postgrado y el doctorado están vinculados directamente a la propia tarea de investigación.

\title{
Ya existe definida una cultura humanista... se puede decir lo mismo en ciencia y tecnología?
}

Esta área debe desarrollarse, pero no solamente en la Universidad de Salamanca, sino que en todas, es decir, la cultura de la ciencia y la tecnología no es un tema de un día para otro, y no vale decir que por tener muchas cosas técnicas exista. Todo lo anterior es un elemento añadido. Tampoco debe haber una dicotomía entre lo que es que uno tenga cultura en ciencia y tecnología y otra persona la tiene en humanidades; hay que buscar elementos de confluencia.

\section{¿Qué importancia reviste la interfaz que representa la comunicación científica en un mundo donde el avance en esta área es avasallador y en el que la sociedad en su conjunto debe ser informada del acontecer científico-tecnológico?}

La prueba de la importancia que le otorga la USAL está, por ejemplo, en el posgrado de Ciencia, Tecnología y Sociedad, incluido en Títulos Propios. Como institución hemos cruzado los muros de la misma universidad, para entrar en contacto con la misma comunidad, pero también dirigiendo la mirada hacia adentro. Es decir, que se ha superado esa cultura que caracterizaba a la universidad, de mantener muchas de las cosas realizadas, sin que trasvasen sus muros. La comunidad universitaria, con sus reglas y sus logros debe mirar hacia fuera sin dejar de atender el funcionamiento interno. 


\section{¿Es capaz de crear la Universidad alguna instancia que fomente la divulgación científica tan necesaria en estos días?}

Considero que ese es uno de los elementos que debe ser trabajado con mayor intensidad. Si evalúo críticamente la ejecución del programa electoral que presentamos en 1998, de hecho, una parte a mejorar sería ésa. Durante la gestión rectoral existieron las posibilidades para hacer esta difusión, pero luego por razones coyunturales no se pudieron llevar a cabo.

\section{El museo de ciencias, por ejemplo. ¿Todavía está pendiente?}

En el caso particular del museo de ciencias ocurrió lo de siempre. $\mathrm{Al}$ final, la universidad, como cualquier instancia que gestiona un presupuesto, tiene que someterse a la economía de aplicar recursos escasos. Entonces surge la pregunta: ¿qué compras, libros o edificas un museo de ciencias? La clave es no entrar en esa opción, porque tampoco es $\mathrm{A}$ o B, es A y B. Lo que pasa es que muchas veces te falta presupuesto y también son tus incapacidades o tu falta de seducción personal para implicar a instancias terceras en todos los proyectos que se quieran hacer.

La tradición del mecenazgo en España no está muy consolidada y yo creo que hay que trabajar también en esa línea.

\section{¿Cómo reflejan los medios de comunicación masiva las actividades llevadas a cabo desde su gestión? ¿Puede esto de alguna forma incidir en un incremento del presupuesto que otorga la administración central?}

Hay una presencia real de los medios de comunicación en relación a las actividades desarrolladas por la Universidad de Salamanca. Que yo, luego, pueda discrepar o estar más o menos de acuerdo con una noticia, es otro asunto. Genéricamente puedo decir que sí, que estoy conforme con la cobertura.

Respecto a lo segundo, creo que es evidente que si se da a conocer lo que haces puedes conseguir financiación externa, esta es una fase que no debe ser pasada por alto. La difusión de nuestro trabajo ayuda, sin duda, a todo lo que sea proyección y captación de recursos. 
La apuesta científica..., entrevista con el Rector

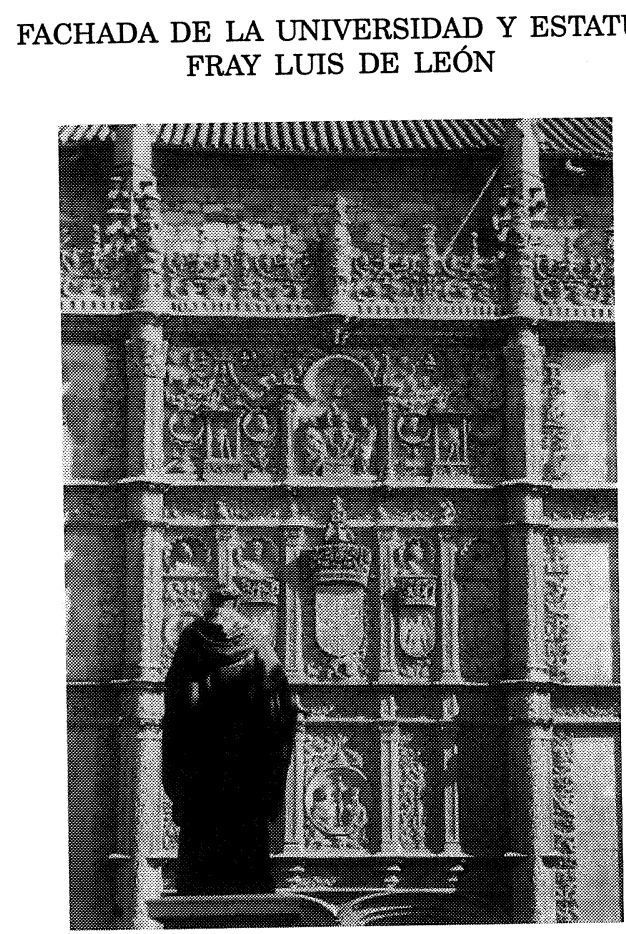

RECTOR DE LA UNIVERSIDAD DE SALAMANCA

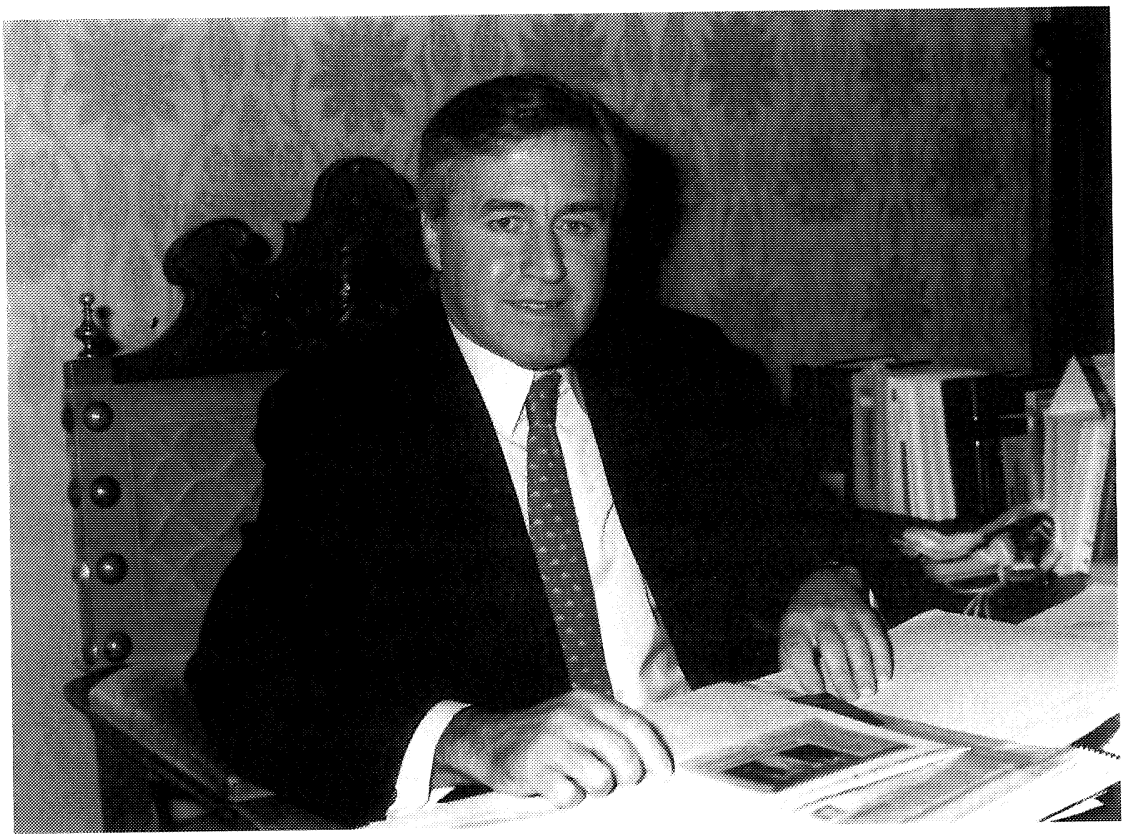

